\title{
Higher-Spin Triplet Fields and String Theory
}

\author{
D. Sorokin
}

\begin{abstract}
We review basic properties of reducible higher-spin multiplets, called triplets, and demonstrate how they naturally appear as part of the spectrum of String Field Theory in the tensionless limit. We show how in the frame-like formulation the triplet fields are endowed with the geometrical meaning of being components of higher-spin vielbeins and connections and present actions describing their free dynamics.
\end{abstract}

\section{Introduction}

It is a pleasure to write this contribution on the occasion of the anniversary of Jiri Niederle. As a topic I have chosen a subject to which Professor Niederle and his collaborators have contributed with several interesting papers $[1,2,3]$. It concerns higher spin field theory. In particular, I would like to discuss a system of higher spin fields which are called triplets. The physical states of triplet fields describe massless particles of decreasing spins. In the bosonic case the physical states of a triplet have spins $s, s-2, s-4, \ldots, 1$ or 0 and the physical states of a fermionic triplet have spin $s, s-1, s-2, \ldots, 1 / 2$. In other words the triplets form reducible Poincaré group multiplets of massless higher-spin particles. As we shall see, they naturally arise in a tensionless limit of String Field Theory $[4,5,6,7,8,9,10]$ as sets of three tensor fields (and this is where their name comes from [8]). We shall also discuss basic group-theoretical and geometrical properties of the higher-spin triplets revealed in [11].

Let us start with a brief generic discussion of problems of Higher Spin Field Theory. The construction of a consistent interacting theory of higher-spin fields is one of the oldest long standing problems in theoretical physics of particles and fields. Since the early 1930s, it has been addressed by many distinguished theorists including Majorana, Dirac, Fierz \& Pauli, Rarita \& Schwinger, Bargmann \& Wigner, Fang \& Fronsdal, Weinberg, Velo \& Zwanziger, Aragon \& Deser, Singh \& Hagen, de Wit \& Freedman, Fradkin \& Vasiliev and (later on) by many others ${ }^{1}$.

The main problem of Higher Spin Field Theory is the construction of consistent interactions of higherspin fields. Higher-spin interactions, e.g. with electromagnetic field and gravity, must respect gauge symmetries and be consistent with quantum mechanical principals such as unitarity, causality, etc.

To solve the problem of higher-spin interactions one first looks for a most appropriate description of free higher-spin fields which would allow for general- ization to an interacting theory. Revealing the grouptheoretical and geometrical structures underlying this theory is of great importance. Until now, the most successful general approach to the construction of nonlinear (i.e. interacting) classical higher-spin field equations has been the so-called unfolding techniques put forward by M. Vasiliev [18, 19] (see [12] for a review and references).

Great efforts have been made in studying both, massive and massless, higher-spin field theories. Jiri Niederle has concentrated, in particular, on studying electro-magnetic interactions of massive higherspin fields, which, actually, reveal major issues of the generic problem $[2,3]$.

It would be very interesting and very important for the further development of this subject to compare the results obtained in the construction of higher-spin interactions with the structure of a known example of consistent higher-spin field theory which is String Theory $^{2}$. People have worked in this direction since the early years of String Theory, and this is how, for example, the system of higher spin triplet fields was first found [4]. So let us make a very short overview of the place of the higher-spin fields in String Theory.

\section{String Theory as a theory of interacting massive higher-spin fields}

String excitations give rise to an infinite number of fields of increasing spin and mass, the mass of a string state being a linear function of its spin (Regge trajectory) with the proportionality coefficient being the string tension $T$. For instance, in open string theory we have

$$
M_{s}^{2} \sim T(s-1)
$$

The infinite tower of higher-spin string states plays a crucial role in ensuring a smooth ultraviolet behavior (or even UV finiteness) of superstring theory, thus making it a consistent theory of quantum gravity, since gravity is an intrinsic part of String Theory.

\footnotetext{
${ }^{1}$ For reviews on various aspects of Higher Spin Field Theory and references see e.g. [12, 13, 14, 15, 16, 17].

${ }^{2}$ Let us recall that String Theory emerged as a proposal to explain (among other things) the mass-spin dependence (known as Regge trajectory) of higher-spin hadronic resonances observed in experiments in the 1960s.
} 
In String Theory the higher-spin excitations are massive, but our experience in the quantum field theory of vector fields teaches us that for quantum consistency the mass of the vector fields should be generated as a result of spontaneous breaking of a symmetry of massless vector fields. If one tries to extrapolate this statement to String Theory, then the natural question arises whether String Theory can be a spontaneously broken phase of an underlying gauge theory of massless higher-spin fields. From eq. (2.1) we see that the mass tends to zero in the limit in which the string tension goes to zero. So people have tried to answer this question by taking a tensionless limit of String Theory (see, e.g. [20, 21, 22, 23] for various aspects of the tensionless string limit and higher-spin theory).

One can try to deduce the structure of higher-spin field interactions from the action of String Field Theory. E.g. the action of Open String Field Theory has the following schematic Chern-Simons-like form (see [24] for more details)

$$
S_{\text {open string }}=\langle\Phi|Q| \Phi\rangle+|\Phi\rangle^{3}
$$

where $|\Phi\rangle$ is a string field and $Q$ is a BRST operator associated with the symmetries of String Theory.

Due to the complexity of the problem, only terms in the Lagrangian describing a system of free massless higher-spin fields have been obtained from the String Field Theory action so far. As we have already mentioned, this system of massless fields was first obtained by S. Ouvry and J. Stern in 1986 [4]. The simplest triplet consists of a symmetric tensor or (in the case of half integer spins) tensor-spinor fields of rank $s, s-1$ and $s-2$

$$
\phi_{m_{1} \cdots m_{s}}(x), \quad C_{m_{1} \cdots m_{s-1}}(x), \quad D_{m_{1} \cdots m_{s-2}}(x)
$$

where the indices $m=0,1, \ldots, D-1$ are indices of space-time of dimension $D$. The spectrum of the physical states of these fields consists of particles of decreasing spin:

$s, s-2, s-4, \ldots, 1$ or $0-$ in the case of bosons

$s, s-1, s-2, \ldots, 1 / 2-$ in the case of fermions.

Since each value of spin corresponds to an irreducible representation of the Poincaré group, the triplet of the fields (2.3) describes a reducible multiplet of higherspin states.

\section{Massless higher-spin triplets from String Theory}

Let us consider how the equations of motion of the triplet fields (2.3) arise in the tensionless and free field limit of String Field Theory. In the tensionless limit $T \rightarrow 0$, the nilpotent open bosonic string BRST operator is (see e.g. [9])

$$
\begin{aligned}
Q & =c_{0} p_{m} p^{m}+\sum_{k \neq 0}\left(c_{-k} p_{m} a_{k}^{m}-\frac{k}{2} b_{0} c_{-k} c_{k}\right), \\
k & = \pm 1, \pm 2, \ldots, \pm \infty \quad Q^{2}=0
\end{aligned}
$$

where $p_{m}$ is the momentum of string center-of-mass, $a_{k}^{m}$ are string creation (for $k<0$ ) and annihilation (for $k>0$ ) oscillator operators (with $|k|=1,2, \ldots, \infty$ labelling the Regge trajectories), $c_{0}$ and $c_{k}$ are string reparametrizaition ghosts, and $b_{0}$ and $b_{k}$ are antighosts. The (anti)commutator relations satisfied by the operators are

$$
\left[a_{k}^{m}, a_{l}^{n}\right]=k \delta_{l+k, 0} \eta^{m n}, \quad\left\{c_{k}, b_{l}\right\}=\delta_{k+l, 0} .
$$

A string field is a sum of an infinite number of higherspin fields generated by acting on the string vacuum with all possible combinations of creation operators

$$
\begin{aligned}
|\Phi\rangle= & \sum_{a^{m} a^{n} \cdots a^{p} \cdots a^{q} c \cdots b \cdots b|0\rangle .} \phi_{m n \cdots p \cdots q \cdots}(x) \cdot
\end{aligned}
$$

Now, one may ask the question, do independent finite subsets of higher-spin fields exist inside (3.6) which satisfy (at least linearized) string field equations of motion that follow from the action (2.2)? The linear field equations are

$$
Q|\Phi\rangle=0
$$

Because of the nilpotency of the BRST operator, they are invariant under the BRST gauge transformations

$$
\delta|\Phi\rangle=Q|\Lambda\rangle, \quad Q^{2}=0 .
$$

To extract from (3.6) a finite independent set of fields satisfying eq. (3.7) let us pick up string states corresponding to a single Regge trajectory (e.g. $\mathrm{k}=1$ ) and cut this trajectory at a level of spin s. Then it turns out that the string field (3.6) reduces and splits into independent pieces each of which is the sum of three terms

$$
\begin{aligned}
|\Phi\rangle_{\text {triplet }}= & \phi_{m_{1} \cdots m_{s}}(x) a_{-1}^{m_{1}} \cdots a_{-1}^{m_{s}}|0\rangle+ \\
& C_{m_{1} \cdots m_{s-1}}(x) a_{-1}^{m_{1}} \cdots a_{-1}^{m_{s-1}} c_{0} b_{-1}|0\rangle+ \\
& D_{m_{1} \cdots m_{s-2}}(x) a_{-1}^{m_{1}} \cdots a_{-1}^{m_{s-2}} c_{-1} b_{-1}|0\rangle .
\end{aligned}
$$

By independent we mean that each set of fields (3.9) independently satisfies the string field equation (3.7). Symmetric tensor fields $\phi_{s}(x), C_{s-1}(x)$ and $D_{s-2}(x)$ form the simplest bosonic higher-spin triplet which satisfies entangled equations of motion that follow from (3.7), namely:

$$
\begin{aligned}
\square \phi_{m_{1} \cdots m_{s}}= & s \partial_{\left(m_{s}\right.} C_{\left.m_{1} \cdots m_{s-1}\right)}, \quad \square \equiv \partial_{n} \partial^{n} \\
C_{m_{1} \cdots m_{s-1}}= & \partial^{n} \phi_{n m_{1} \cdots m_{s-1}}-(s-1) . \\
& \partial_{\left(m_{s-1}\right.} D_{\left.m_{1} \cdots m_{s-2}\right)}, \\
\square D_{m_{1} \cdots m_{s-2}}= & s \partial^{n} C_{n m_{1} \cdots m_{s-2}},
\end{aligned}
$$


where $\left(m_{1} \cdots m_{p}\right)$ denotes the symmetrization of the indices with weight $\frac{1}{p !}$.

Equations (3.10) are invariant under the gauge transformations with a local symmetric parameter $\Lambda_{m_{1} \cdots m_{s-1}}(x)$ that follow from the BRST symmetry (3.8):

$$
\begin{aligned}
\delta \phi_{m_{1} \cdots m_{s}} & =s \partial_{\left(m_{s}\right.} \Lambda_{\left.m_{1} \cdots m_{s-1}\right)} \\
\delta C_{m_{1} \cdots m_{s-1}} & =\square \Lambda_{m_{1} \cdots m_{s-1}} \\
\delta D_{m_{1} \cdots m_{s-2}} & =\partial^{n} \Lambda_{n m_{1} \cdots m_{s-2}} .
\end{aligned}
$$

From the second of eqs. (3.10) we see that $C_{s-1}$ is an auxiliary field which is expressed in terms of derivatives of $\phi_{s}$ and $D_{s-1}$, and from the form of the gauge variation of $D_{s-1}$ we see that this field is a pure gauge. Thus, all physical degrees of freedom are contained in the field $\phi_{m_{1} \cdots m_{s}}(x)$. As we have already mentioned and as follows from the analysis of eqs. (3.10)-(3.11) [9], the physical degrees of freedom of $\phi_{m_{1} \cdots m_{s}}(x)$ are particle states of $\operatorname{spin} s, s-2, s-4, \ldots, 1$ or 0 (depending whether $s$ is even or odd).

A question which can now be addressed is whether the triplet fields may have some geometrical nature?

\section{Geometrical meaning of HS triplet fields. Frame-like formulation}

Higher Spin Field Theory is a gauge theory. Understanding its underlying group-theoretical and geometrical structure is of great importance for making progress in solving the higher-spin interaction problem. Understanding the geometrical nature of the triplets is part of this generic problem. As far as the physical field $\phi_{m_{1} \cdots m_{s}}(x)$ is concerned, its grouptheoretical properties resemble very much the metric field of General Relativity. The gravitational field $g_{m n}(x)$ is symmetric and it transforms under linearized diffeomorphisms as a gauge field

$$
\delta g_{m n}=\partial_{m} \xi_{n}(x)+\partial_{n} \xi_{m}(x),
$$

which is similar to the transformation properties (3.11) of $\phi_{m_{1} \cdots m_{s}}(x)$.

What about auxiliary fields $C_{s-1}(x)$ and $D_{s-2}(x)$ ? Can they be related to other geometrical quantities, such as a generalized Christoffel symbol, i.e. a higherspin connection associated with the higher-spin local symmetry? The answer to this question is positive. The geometrical nature of the triplet fields manifests itself rather clearly in the frame-like formulation [11] which is the generalization to higher-spin fields $[25,26]$ of the Cartan-Tamm-Weyl formulation of gravity in terms of vielbeins and spin connections.

In the frame formulation of gravity the gravitational field is described by a vielbein one-form

$$
e^{a}=d x^{m} e_{m}^{a}(x),
$$

which carries the tangent space Lorentz index. In this formulation, in addition to the local diffeomorphisms, the theory of gravity possesses local Lorentz symmetry

$$
\delta e^{a}(x)=e^{b} \xi_{b}^{a}(x)
$$

where $\xi_{a b}=-\xi_{b a}$ is a parameter of local Lorentz transformation. The gauge field associated with local Lorentz symmetry is the spin connection

$$
\omega^{a b}=d x^{m} \omega_{m}^{a b}(x)=-\omega^{b a},
$$

which transforms under the infinitesimal local Lorentz transformations as follows

$$
\delta \omega^{a b}=d \xi^{a b}-\xi^{a}{ }_{c} \omega^{c b}+\xi^{b}{ }_{c} \omega^{c a} .
$$

The metric field $g_{m n}$ is composed of the vielbeins

$$
g_{m n}=e_{m}^{a} e_{n}^{b} \eta_{a b}
$$

In the linear approximation, in which $e_{m}{ }^{a}=\delta_{m}^{a}+$ $\tilde{e}_{m}{ }^{a}(x)$ and $g_{m n}=\eta_{m n}+\tilde{g}_{m n}(x)$, eq. (4.16) reduces to

$$
\tilde{g}_{a b}=\tilde{e}_{a b}+\tilde{e}_{b a} .
$$

Note that in the linear approximation there is no distinction between world indices $m, n, \ldots$ and tangent space indices $a, b, \ldots$

The Einstein gravity is characterized by the socalled torsion-free constraint which relates the vielbein and spin connection

$$
d e^{a}+e^{b} \omega_{b}^{a}=0 .
$$

By analogy with the frame formulation of gravity, in the frame-like formulation of higher-spin field theory $[25,26]$ one introduces a higher-spin vielbein one-form, which is symmetric in the $s-1$ tangent-space indices,

$$
e^{a_{1} \cdots a_{s-1}}=d x^{m} e_{m}^{a_{1} \cdots a_{s-1}}
$$

and the higher-spin connection

$$
\omega^{a_{1} \cdots a_{s-1}, b}=d x^{m} \omega_{m}^{a_{1} \cdots a_{s-1}, b}
$$

which is symmetric in the indices $a_{1} \cdots a_{s-1}$ and satisfies the following properties: i) the symmetrization of all of its tangent-space indices $a$ and $b$ brings zero

$$
\omega^{\left(a_{1} \cdots a_{s-1}, b\right)}=0
$$

and ii) the trace of the index $b$ with any of the indices $a$ is zero

$$
\omega^{a_{1} \cdots a_{s-1}, b} \eta_{a_{1} b}=0 .
$$

The properties (4.21) and (4.22) of the higher-spin connection are somewhat analogous to the antisymmetry property of the spin connection (4.20). To describe single higher-spin fields, one should impose on the higherspin vielbein and connection stronger trace-less conditions (see $[12,11]$ for a review and references)

$$
\begin{aligned}
& e^{a_{1} a_{2} \cdots a_{s-1}} \eta_{a_{1} a_{2}}=0, \\
& \omega^{a_{1} a_{2} \cdots a_{s-1} b}, \eta_{a_{1} a_{2}}=0 \text {. }
\end{aligned}
$$


If eqs. (4.23) are satisfied, then the traceless condition (4.22) for $\omega$ follows from (4.23) and (4.21). As has been shown in [11], the reducible triplet multiplets are described by $e$ and $\omega$ which are not subject to the traceless constraints (4.23) and $\omega$ satisfies a less restrictive constraint (4.22).

As in the case of Einstein gravity, we also assume that the higher-spin vielbein and connection satisfy the torsion-free condition

$$
d e^{a_{1} \cdots a_{s-1}}-d x^{m} \omega^{a_{1} \cdots a_{s-1}, b} \eta_{m b}=0 .
$$

The torsion-free condition is preserved under the following variation of $e$ and $\omega$, which are local gauge symmetries of the frame-like formulation

$$
\begin{aligned}
& \delta e^{a_{1} \cdots a_{s-1}}= \\
& d \xi^{a_{1} \cdots a_{s-1}}(x)-(s-1) \xi^{a_{1} \cdots a_{s-1}, b}(x) \eta_{m b}, \\
& \delta \omega^{a_{1} \cdots a_{s-1}, b}= \\
& d \xi^{a_{1} \cdots a_{s-1}, b}(x)-(s-2) \xi^{a_{1} \cdots a_{s-1}, b c}(x) \eta_{m c}
\end{aligned}
$$

where the parameter $\xi^{a_{1} \cdots a_{s-1}}$ is symmetric, the parameter $\xi^{a_{1} \cdots a_{s-1}, b}(x)$ has the same symmetry properties as $\omega$ and the parameter $\xi^{a_{1} \cdots a_{s-1}, b c}$ is symmetric in the indices $a_{1} \cdots a_{s-1}$ and (separately) in the indices $b c$. In addition, the symmetrization in $\xi^{a_{1} \cdots a_{s-1}, b c}$ of either $b$, or $c$ with all $a$ gives zero, and the trace of $b$ or $c$ with any $a$ also gives zero.

The higher-spin triplet fields (3.9) turn out to be components of the higher-spin vielbein and connection [11]. The relation is as follows

$$
\begin{aligned}
\phi^{a_{1} \cdots a_{s}} & =e_{m}{ }^{\left(a_{1} \cdots a_{s-1}\right.} \eta^{\left.a_{s}\right) m}, \\
D^{a_{1} \cdots a_{s-2}} & =e_{m}^{{ }^{a_{1} \cdots a_{s-2} a_{s-1}} \delta_{a_{s-1}}^{m}} \\
C^{a_{1} \cdots a_{s-1}} & =(s-1) \omega_{m}^{a_{1} \cdots a_{s-1}, m}+\partial^{m} e_{m}^{a_{1} \cdots a_{s-1}} .
\end{aligned}
$$

The relation (4.26) between the two sets of fields has been established in [11] by comparing their gauge transformations and equations of motion in the framelike and metric-like formulation. In the frame-like formulation, for the higher-spin vielbein and connection the equations of motion are the torsion-free condition (4.24), which allows one to express the components of $\omega$ in terms of derivatives of $e$, up to the Stueckelberg gauge transformations with the parameter $\xi^{a_{1} \cdots a_{s-1}, b c}(x)$ of eq. (4.25), and the following differential equation on $\omega$

$$
\begin{aligned}
& \delta_{(b}^{m} \partial^{c} \omega^{d ;}{ }_{\left.n_{1} \cdots n_{s-2}\right)[c, d]}+\partial_{d} \omega_{\left(b ; n_{1} \cdots n_{s-2}\right)}{ }^{[m, d]}+ \\
& \partial_{(b} \omega^{d ;}{ }_{\left.n_{1} \cdots n_{s-2}\right)[d}{ }^{m]}=0,
\end{aligned}
$$

where the indices separated by ';' are world indices, i.e. they correspond to the index $m$ in (4.20). This equation is the dynamical field equation of the higherspin vielbein, when $\omega$ is expressed in terms of $\partial e$ in virtue of the torsion-free conditions.

The equations of motion (4.24) and (4.27) can be obtained from the action which generalizes to higherspin fields the action for gravity in the frame formulation. In any dimension $D>1$ the gravity action can be written as an integral over the differential form

$$
\begin{aligned}
& S\left[e^{a}, \omega^{a b}\right]=\frac{1}{2} \int_{\mathcal{M}^{D}} e^{a_{1}} \ldots e^{a_{D-2}} \varepsilon_{a_{1} \ldots a_{D-2} b c} R^{b c}= \\
& \frac{1}{2} \int_{\mathcal{M}^{D}} e^{a_{1}} \ldots e^{a_{D-2}} \varepsilon_{a_{1} \ldots a_{D-2} b c}\left(d \omega^{b c}+\omega^{b}{ }_{d} \omega^{d c}\right),
\end{aligned}
$$

or up to a total derivative

$$
\begin{aligned}
& S\left[e^{a}, \omega^{a b}\right]=\frac{1}{2} \int_{\mathcal{M}^{D}} \varepsilon_{a_{1} \ldots a_{D-3} d b c} e^{a_{1}} \ldots e^{a_{D-3}} \\
& \left((D-2) d e^{d} \omega^{b c}+e^{d} \omega_{f}^{b} \omega^{f c}\right)= \\
& \frac{D-2}{2} \int_{\mathcal{M}^{D}} \varepsilon_{a_{1} \ldots a_{D-3} b c d} e^{a_{1}} \ldots e^{a_{D-3}} . \\
& \left(d e^{b} \omega^{c d}-\frac{1}{2} e^{f} \omega_{f}^{b}\right) \omega^{c d}
\end{aligned}
$$

where the wedge product of the differential forms is assumed.

The higher-spin generalization of the linearized gravity action (4.29) in Minkowski space is

$$
\begin{aligned}
& S=\int_{M^{D}} d x^{a_{1}} \ldots d x^{a_{D-3}} \varepsilon_{a_{1} \ldots a_{D-3} p q r} . \\
& \left(d e^{n_{1} \ldots n_{s-2} p}-\frac{s-1}{2} d x_{m} \omega^{n_{1} \ldots n_{s-2} p, m}\right) \omega_{n_{1} \ldots n_{s-2}}{ }^{q, r} .
\end{aligned}
$$

This action is a straightforward generalization of the four-dimensional action of [25]. The torsion-free condition (4.24) and the dynamical equation (4.27) are obtained by varying eq. (4.30) with respect to $\omega$ and $e$, respectively.

Let us recall that if in equations (4.24), (4.27) and (4.30) the higher-spin vielbein and connection together with corresponding gauge symmetry parameters were subject to the additional traceless condition in their symmetric target-space indices (4.23), the frame-like formulation based on the action (4.30) would describe irreducible physical states corresponding to massless particles of single spin $s$ (see [11] for more details). It is a certain relaxation of the trace constraints on the higher-spin vielbeins and connections which extended the irreducible higher-spin system to the reducible (triplet) higher-spin multiplet. It should also be noted that because of the peculiarity of the form of the frame-like action (4.30), which is constructed of the wedge product of one-forms $\omega$ and (the differential) of $e$, it describes the higher-spin triplets whose physical states have spins $s, s-1, \ldots, 3$ or 2 . The scalar and the vector fields can nevertheless be added to this construction in a conventional way (see $[12,11]$ for more discussion of this point).

An analogous formulation has been constructed in [11] for the fermionic higher-spin triplets describing physical states of $\operatorname{spin} s, s-1, \ldots, 3 / 2$. As in the bosonic case regarding the scalar and the vector states 
of the triplets, the field of spin $1 / 2$ can be included into this construction as an independent field.

The action for the fermionic triplets is of the first order in derivatives of the fermionic one-form

$$
\psi^{a_{1} \cdots a_{s-1 / 2}, \alpha}=d x^{m} \psi_{m}^{a_{1} \cdots a_{s-1 / 2}, \alpha},
$$

where $\alpha$ is the spinorial index and $s$ is now a halfinteger. The field $\psi$ is symmetric in the indices $a$. In a $D$-dimensional flat space-time the fermionic triplet action in the frame-like formulation has a very simple form

$$
\begin{aligned}
S= & i \int_{M^{D}} \mathrm{~d} x^{a_{1}} \ldots \mathrm{d} x^{a_{D-3}} \varepsilon_{a_{1} \ldots a_{D-3} p q r} . \\
& \left(\bar{\psi}_{d_{1} \ldots d_{s-\frac{3}{2}}} \gamma^{p q r} d \psi^{d_{1} \ldots d_{s-\frac{3}{2}}}-\right. \\
& \left.6\left(s-\frac{3}{2}\right) \bar{\psi}_{d_{1} \ldots d_{s-\frac{5}{2}}}{ }^{p} \gamma^{q} d \psi^{d_{1} \ldots d_{s-\frac{5}{2} r}}\right),
\end{aligned}
$$

It is invariant under the following local transformations of the field $\psi$

$$
\begin{aligned}
& \delta \psi_{a_{1} \ldots a_{s-\frac{3}{2}}}= \\
& \mathrm{d} \xi_{a_{1} \ldots a_{s-\frac{3}{2}}}-\left(s-\frac{3}{2}\right) \mathrm{d} x^{b} \xi_{a_{1} \ldots a_{s-\frac{3}{2}}, b}
\end{aligned}
$$

with the tensor-spinor parameter $\xi_{a_{1} \ldots a_{s-\frac{3}{2}}, b}^{\alpha}$ having the following symmetry properties

$$
\begin{aligned}
\xi_{a_{1} \ldots a_{s-\frac{3}{2}}, b} & =\xi_{\left(a_{1} \ldots a_{s-\frac{3}{2}}\right), b}, \\
\xi_{\left(a_{1} \ldots a_{s-\frac{3}{2}}, b\right)} & =0
\end{aligned}
$$

and being subject to the (gamma)-traceless constraints with respect to the index $b$

$$
\gamma_{b} \xi^{a_{1} \ldots a_{s-\frac{3}{2}}, b}=0 \quad \xi^{a_{1} \ldots a_{s-\frac{5}{2}} b}{ }_{b}=0 .
$$

If the fermionic one-form $\psi$ and the fermionic parameters of the gauge transformations (4.33) were (in addition) gamma-traceless in the indices $a$, the action (4.32) would describe a single (irreducible) higher-spin field of spin $s$.

The frame-like formulation of the bosonic and fermionic higher-spin triplet fields considered above can be generalized to describe these fields in the antide Sitter space [11]. In particular, the use of the framelike formulation has allowed us to overcome technical difficulties encountered in $[9,27]$ and to obtain in [11] a description of the fermionic higher-spin triplets in AdS. This is a first step towards the study of consistent interactions of fermionic triplets, since, as has been known for a long time, a consistent theory of interacting massless higher-spin fields should be formulated in a space-time background of constant curvature, like the AdS space.

\section{Conclusion}

We have seen how a triplet system of fields of spin $s, s-2 \ldots, 1$ or 0 appears in a truncated action for String Field Theory. Using the frame-like formulation, we endowed these fields with a geometrical meaning of higher-spin vielbeins and connections subject to a torsion-free condition and transforming under higherspin local symmetries. The frame-like actions for the bosonic and fermionic triplet fields have been constructed in flat and AdS spaces. It is of great interest and importance to generalize these results by analyzing possible interactions of the higher-spin triplet fields (see $[17,28]$ for a recent discussion of this issue and references). This would give a new insight into the structure of Higher Spin Field Theory, String Theory and AdS/CFT correspondence.

\section{Acknowledgement}

This work was partially supported by the INFN Special Initiative TV12, the INTAS Project Grant 051000008-7928, and an Excellence Grant of Fondazione Cariparo.

\section{References}

[1] Kotecky, R., Niederle, J.: Conformally Covariant Field Equations II. First Order Massless Equations, Rept. Math. Phys. 12 (1977) 237-249.

[2] Niederle, J., Nikitin, A. G.: Relativistic wave equations for interacting massive particles with arbitrary half-integer spins, Phys. Rev. D64 (2001) 125013.

[3] Niederle, J., Nikitin, A. G.: Relativistic Coulomb problem for particles with arbitrary half-integer spin, J. Phys. A39 (2006) 10 931-10944, arXiv:hep-th/0412214.

[4] Ouvry, S., Stern, J.: Gauge fields of any spin and symmetry, Phys. Lett. B177 (1986) 335.

[5] Bengtsson, A. K. H.: A unified action for higher spin gauge bosons from covariant string theory, Phys. Lett. B182 (1986) 321.

[6] Henneaux, M., Teitelboim, C.: First and second quantized point particles of any spin. In Santiago 1987, Proceedings, Quantum mechanics of fundamental systems 2 113-152. (see Conference Index).

[7] Pashnev, A. I.: Composite systems and field theory for a free Regge trajectory, Theor. Math. Phys. 78 (1989) 272-277.

[8] Francia, D., Sagnotti, A.: On the geometry of higher-spin gauge fields, Class. Quant. Grav. 20 (2003) S473-S486. 
[9] Sagnotti, A., Tsulaia, M.: On higher spins and the tensionless limit of string theory, Nucl. Phys. B682 (2004) 83-116.

[10] Barnich, G., Bonelli, G., Grigoriev, M.: From BRST to light-cone description of higher spin gauge fields, arXiv: hep-th/0502232.

[11] Sorokin, D. P., Vasiliev, M. A.: Reducible higherspin multiplets in flat and AdS spaces and their geometric frame-like formulation, Nucl. Phys. B809 (2009) 110-157.

[12] Bekaert, X., Cnockaert, S., Iazeolla, C., Vasiliev, M. A.: Nonlinear higher spin theories in various dimensions, arXiv:hep-th/0503128.

[13] Vasiliev, M. A.: Higher spin gauge theories in various dimensions, Fortsch. Phys. 52 (2004) $702-717$.

[14] Sorokin, D.: Introduction to the classical theory of higher spins, AIP Conf. Proc. 767 (2005) 172-202, arXiv:hep-th/0405069.

[15] Bouatta, N., Compere, G., Sagnotti, A.: An introduction to free higher-spin fields, arXiv:hep-th/0409068.

[16] Sagnotti, A., Sezgin, E., Sundell, P.: On higher spins with a strong $\operatorname{Sp}(2, \mathrm{R})$ condition, arXiv: hep-th/0501156.

[17] Fotopoulos, A., Tsulaia, M.: Gauge Invariant Lagrangians for Free and Interacting Higher Spin Fields. A Review of the BRST formulation, Int. J. Mod. Phys. A24 (2009) 1-60.

[18] Vasiliev, M. A.: Equations of motion of interacting massless fields of all spins as a free differential algebra, Phys. Lett. B209 (1988) 491-497.

[19] Vasiliev, M. A.: Consistent equations for interacting massless fields of all spins in the first order in curvatures, Annals Phys. 190 (1989) 59-106.
[20] Gross, D. J.: High-Energy Symmetries of String Theory, Phys. Rev. Lett. 60 (1988) 1229.

[21] Sundborg, B.: Stringy gravity, interacting tensionless strings and massless higher spins, Nucl. Phys. Proc. Suppl. 102 (2001) 113-119.

[22] Lindstrom, U., Zabzine, M.: Tensionless strings, WZW models at critical level and massless higher spin fields, Phys. Lett. B584 (2004) 178-185.

[23] Bonelli, G.: On the tensionless limit of bosonic strings, infinite symmetries and higher spins, Nucl. Phys. B669 (2003) 159-172.

[24] Witten, E.: Interacting Field Theory of Open Superstrings, Nucl. Phys. B276 (1986) 291.

[25] Vasiliev, M. A.: 'Gauge' form of description of massless fields with arbitrary spin. (in Russian), Yad. Fiz. 32 (1980) 855-861.

[26] Aragone, C., Deser, S.: Higher spin vierbein gauge fermions and hypergravities, Nucl. Phys. B170 (1980) 329.

[27] Buchbinder, I. L., Galajinsky, A. V., Krykhtin, V. A.: Quartet unconstrained formulation for massless higher spin fields, Nucl. Phys. B779 (2007) 155-177.

[28] Fotopoulos, A., Tsulaia, M.: Current Exchanges for Reducible Higher Spin Multiplets and Gauge Fixing, JHEP 10 (2009) 050.

Dmitri Sorokin

E-mail: dmitri.sorokin@pd.infn.it

INFN, Sezione di Padova

via F. Marzolo 8

35131 Padova, Italia 